\title{
Accident Detection and Message Conveyor System using GSM and GPS Module
}

\author{
Khyati Shah \\ NMIMS MPSTME, Vile Parle, \\ Mumbai, India
}

\author{
Swati Bairagi \\ NMIMS MPSTME, Vile Parle, \\ Mumbai, India
}

\begin{abstract}
With the growing population the use of vehicles has become superfluous and this has led to increase in the number of accidents at an alarming rate. This project aims at detecting the accident and reporting the location of accident to the previously coded numbers. This helps in a speedy service from the ambulance and the concerned person. The GPS and GSM technology is used to locate the position of the vehicle in the form of latitude and longitude coordinates and sends it through SMS.

Piezoelectric and alcohol sensors when triggered, help in detecting the accident and sending the signal to the micro controller of the system. This further helps in sending the message to the concerned person. This system can help companies in the rental vehicle business to keep a track of the vehicular activity by sending message at regular intervals to the authorized numbers.
\end{abstract}

\section{Keywords}

GPS, GSM, Arduino Uno, alcohol and piezoelectric sensors.

\section{INTRODUCTION}

We often come across the fact that when an accident occurs the people nearby have to manually call the ambulance which leads to waste of time. Hence there is a delay for emergency services to arrive at the location of the accident

Proposed system makes an effort to provide the emergency facilities to the victims in the shortest time possible. It incorporates an embedded system that contains GPS and GSM modules connected with an Arduino UNO. The entire set-up is installed at the front end of the vehicle. An alcohol sensor is used to detect the Ethanol level in the breath of the driver. A piezoelectric sensor is used to measure the vibration at the time of accident. Global Positioning System (GPS) is used to identify the location of the vehicle. GSM is used to inform the exact vehicular location in the form of the longitude and latitude coordinates to the precoded numbers using an SMS. GSM module provides a two way communication by using a SIM card. Such a module works in the same manner as a regular phone. This application provides the optimum solution to poor emergency facilities provided to the roads accidents in the most feasible way.

\section{METHODOLOGY}

The main elements of the prototype model of an automatic vehicle accident detection and messaging are GSM and GPS module and Arduino UNO. The working of this model will be made in the following steps:

1. An alcohol sensor is basically used as a breath tester. It senses the Ethanol content in the driver's breath and gives its output to the motor. If the level of the ethanol is beyond the limit then motor stops (brakes) else the motor is $\mathrm{ON}$.

2. A piezoelectric sensor is used to sense the vibration at the time of the accident and give its output to the buzzer. If any value of vibration sensor is changed beyond limit then buzzer will start. If the buzzer is ON for more than 10 seconds, the GPS module is triggered $\mathrm{ON}$. In case of a minor accident, where the driver does not need any external assistance, he/she will press the switch within 10 seconds due to which the buzzer will be OFF and the GPS will not be triggered. The GPS (Global Positioning System) detects the latitude and longitudinal position of a vehicle.

3. The latitudes and longitude position of the vehicle is sent as a message through the Global System for Mobile Communication (GSM) to the pre-saved numbers.

\section{BLOCK DIAGRAM OF THE SYSTEM}

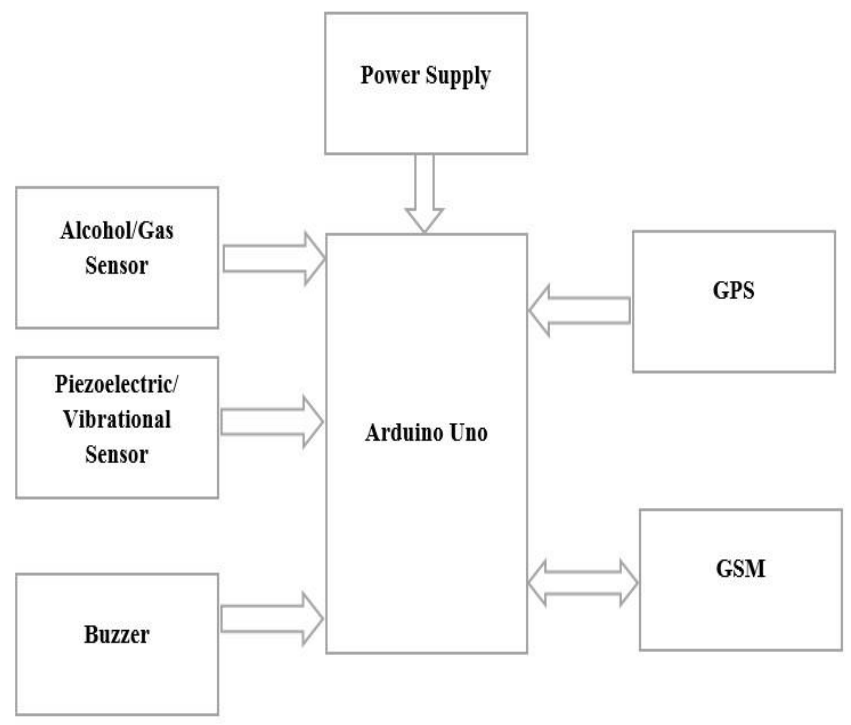

Fig. 3 Proposed Accident Detection System

\section{HARDWARE COMPONENTS AND DESIGN}

\subsection{Arduino UNO}

Arduino Uno is a micro-controller board based on the ATmega328P. It has 14 digital input/output pins, out of which 6 can be used as PWM outputs, 6 analog inputs, a $16 \mathrm{MHz}$ quartz crystal, a USB connection, a power jack, an ICSP header and a reset button [7]. The Arduino Uno can be powered via the USB connection or with an external power 
supply[1]. The power source is selected automatically. The board can operate on an external supply of 6 to 20 volts thought The recommended range is 7 to 12 volts. 3, 5, 6, 9, 10, and 11. Provide 8-bit PWM output with the analogWrite() function. External (non-USB) power can come either from an AC-to-DC adapter (wall-wart) or battery. The adapter can be connected by plugging a $2.1 \mathrm{~mm}$ center-positive plug into the board's power jack. Leads from a battery can be inserted in the Gnd and Vin pin headers of the POWER connector.

\subsection{Alcohol Sensor}

Alcohol sensor typically used as a part of the breathalyses or breath tester for the detection of ethanol in the human breath. MQ-3 gas sensor has high sensitivity and fast response time to alcohol and has good resistance to disturb of gasoline, smoke and vapour. Sensitive material of MQ-3 is $\mathrm{SnO} 2$, which with lower conductivity in clean air. Mainly vapour. Sensitive material of MQ-3 is $\mathrm{SnO}$, which with lower conductivity in clean air. Mainly or. A simple interface could be a 0-3.3V $\mathrm{ADC}$

\subsection{Vibration Sensor}

In this project piezoelectric sensor is used as a vibration sensor. A piezoelectric sensor is a device that uses the piezoelectric effect, to measure changes in pressure, acceleration, temperature, strain, or force by converting them to an electrical charge.

\subsection{Buzzer}

The buzzer used is 1-8S LiPo Battery Voltage Tester Low Volt Alarm Buzzer and LED. It is used for testing 1-8S Lipo/Li-ion/LiMn/Li-Fe. The Voltage Detection Precision is +/- 0.01V. The Unit Voltage Display Range: 0.5 4.5V. The $1 \mathrm{~S}$ Test Mode Voltage Range: $3.7 \sim 30 \mathrm{~V}$ and there is a Low voltage alarm mode for $2-8 \mathrm{~S}$. The Alarm Set Value Range is $\mathrm{OFF} \sim 2.7 \sim 3.8 \mathrm{~V}$.

\subsection{GPS Module}

The GPS used in this project is NEO6mv2.The NEO-6 module series is a family of stand-alone GPS receivers featuring the high performance u-blox 6 positioning engine. These flexible and cost effective receivers offer numerous connectivity engine. These flexible and cost effective receivers offer numerous connectivity deal for battery operated mobile devices with very strict cost and space constraints. The 50-channel u-blox 6 positioning engine boasts a Time-To-First-Fix0(TTFF) of under 1 second[4].The dedicated acquisition engine, with 2 million correctors, is capable of massive parallel time/frequency space searches, enabling it to find satellites instantly. Innovative design and technology suppresses jamming sources and mitigates multipath effects, giving NEO-6 GPS receivers excellent navigation performance even in the most challenging environments. The Supply Voltage is 2.7 to $3.6 \mathrm{~V}$. The Supply current is $67 \mathrm{~mA}$ of antennas in this GPS i.e. Passive and active antenna.

\subsection{GSM Module}

Most modern cellular networks make use of the GSM technology for communication purposes. In this project we are using SIM800 module which works in the frequency band of $800 \mathrm{MHz}$.

\section{ALGORITHM}

\section{Step 1: Start}

Step 2: Vehicle starts and the alcohol sensor gets triggered.

Step 3: If value is greater than 0.3 than end else goto step 4 .

Step 4: Vehicle continues if pressure detected value is smaller the ' 0 '.

Step 5: If pressure detected value is greater than ' 0 ' than buzzer turns on for 10 seconds.

Step 6: If buzzer turned off in 10 seconds then end else goto step 3.

Step 7: GPS detects the exact location on of the vehicle

Step 8: Send text message to the pre-saved numbers.

Step 9: Stop.

\section{FLOW CHART}

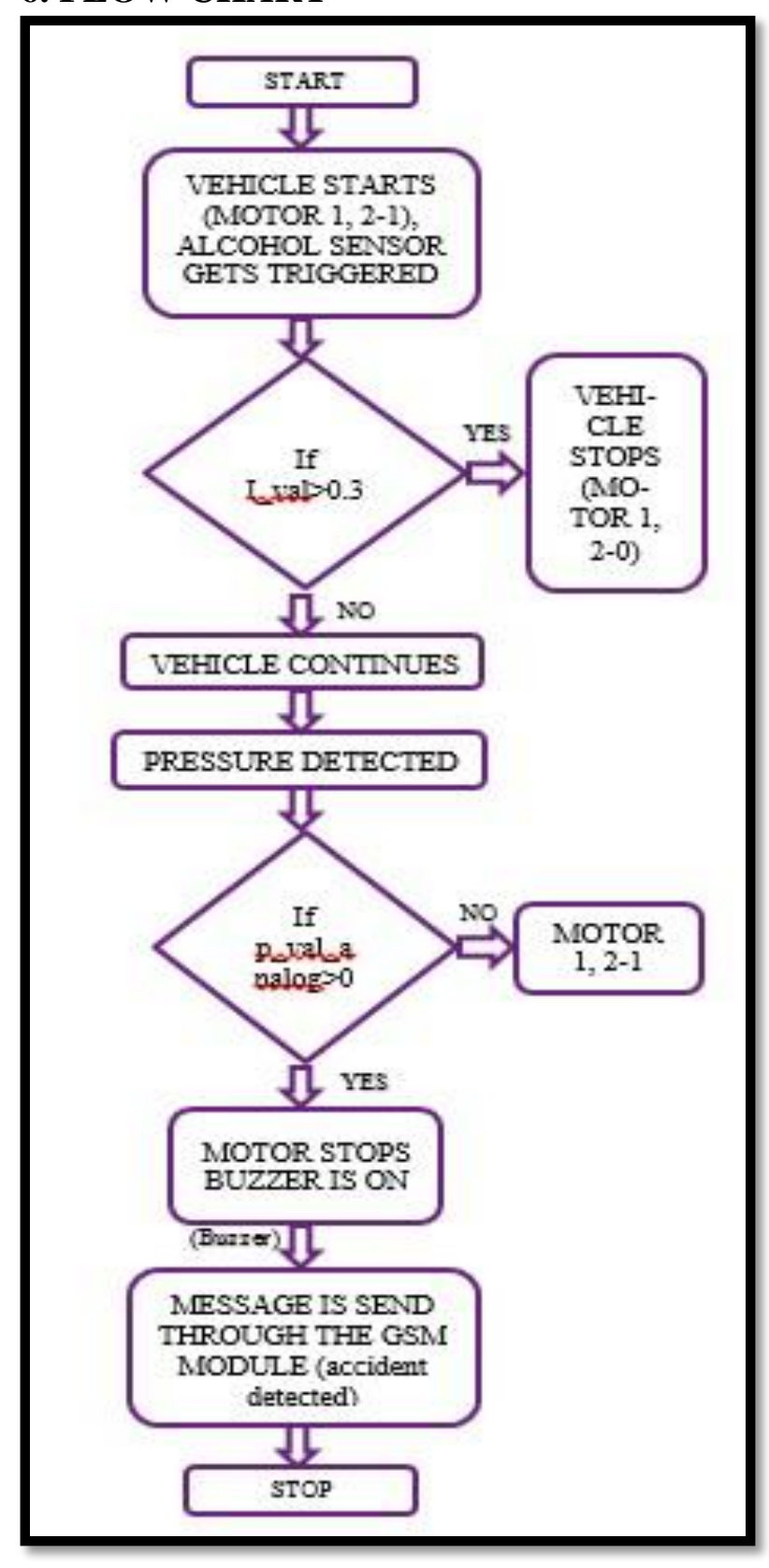

Figure 6.1 Flowchart of Proposed Model 


\section{RESULT}

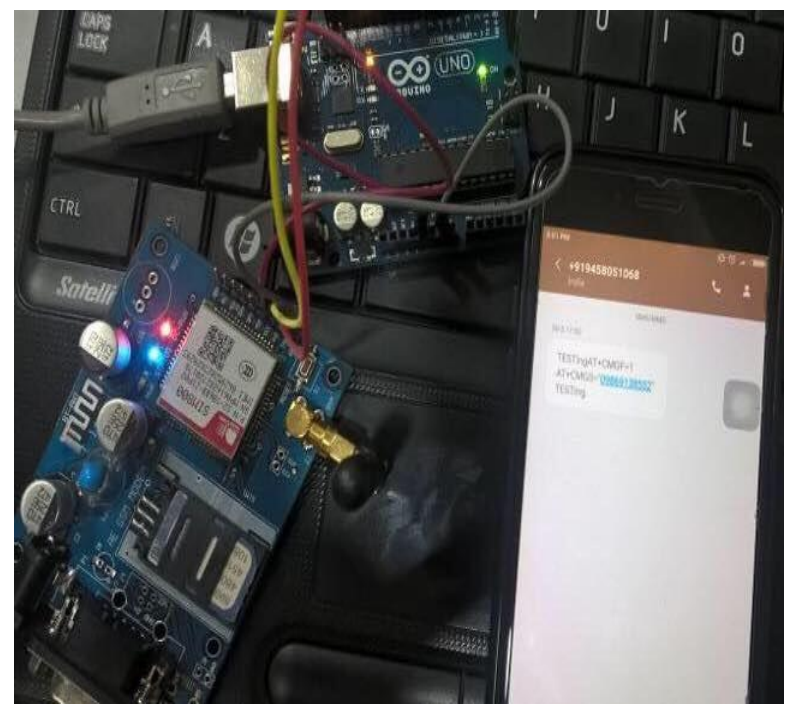

Fig. 7.1 Interfacing of GSM with Arduino

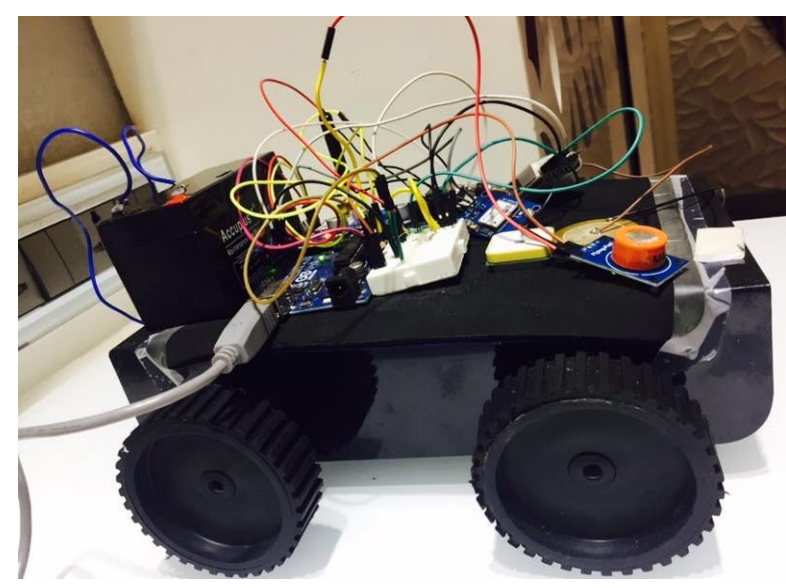

Fig. 7.2 Final System Model

\section{CONCLUSION}

The proposed model for accident detection system can prove to be an important aid in constructing smart transport systems in near future if implemented properly. In this various sensors are placed which indicates occurrence of an accident just like vibration sensor, alcohol sensor. If range of that sensor increases beyond specific range then buzzer will get "on" for ten seconds and driver can't stop the buzzer within ten seconds then it will detect as an accident and a message will send to previously stored numbers with co-ordinates of that location.

\section{REFERENCES}

[1] Sachin M S, Prasanna P, "Automatic vehicle accident detection and traffic control system", International Journal on Recent and Innovation Trends in Computing and Communication, Vol. 3 Issue 6, 2015.

[2] D. Kumar, S. Gupta et. al., "Accident Detection and Reporting System Using GPS and GSM Module", Journal of Emerging Technologies and Innovative Research, Volume 2, Issue 5, 2015.

[3] Sri Krishna C Varma, Poornesh et. al. "Auto matic Vehicle Accident Detection and Messaging System Using GPS and GSM Modem", International Journal of Scientific \& Engineering Research, Volume 4, Issue 8, 2013.

[4] Pratiksha R., Shetgaonkar et. al., "Proposed Model for the Smart Accident Detection System for Smart Vehicles using Arduino board, Smart Sensors, GPS and GSM" International Journal of Emerging Trends \& Technology in Computer Science vol. 4, issue 4, 2015.

[5] The 8051 Microcontroller and Embedded Systems" by Muhammad Ali Mazidi and Janice Gillispie Mazidi, Pearson Education.

[6] "8051 Microcontroller Architecture, programming and application" by KENNETH JAYALA.

[7] ATMEL 89S52 Data sheets.

[8] Wang Wei, Fan Hanbo- "Traffic Accident Automatic Detection And Remote Alarm Device", IEEE , 2011. 